\title{
Aspectos clínico-epidemiológicos de la toxoplasmosis en pacientes que consultan por problemas de visión
}

\author{
Margarita Samudio, M. Eugenia Acosta, Verónica Castillo, Yvalena Guillén, Gizel Licitra, Laura Aria, Pablo Cibils, \\ Alejandra Rojas, Diógenes Cibils, Teresa Meza, Belén Infanzón y Herminia Miño de Kaspar
}

Universidad Nacional Autónoma

Instituto de Investigaciones en Ciencias de la Salud (MS, MEA, YG, $L A, A R, T M, B I)$ Facultad de Ciencias Médicas (VC, $\mathrm{GL}, \mathrm{PC}, \mathrm{DC})$

Cátedra de Oftalmología. Universidad de Munich, Alemania (HMK).

Financiamiento: Fundación Georg Hannelore Zimmermann. Munich-Alemania Conflictos de interés: no hay.

Recibido: 22 de mayo de 2015 Aceptado: 9 de noviembre de 2015

Correspondencia a: Margarita Samudio margarita.samudio@gmail.com

\section{Clinical and epidemiological aspects of toxoplasmosis in patients with vision problems}

Introduction: Toxoplasmosis is a worldwide disease; it can cause decreased vision or even blindness. The route of transmission in humans may vary according to the habits of the region; probably the ingestion of raw or undercooked meat is the main source of infection. Objective: To determine the seroprevalence of toxoplasmosis in an eye clinic, the frequency of ocular toxoplasmosis (OT) and risk habits for acquiring the infection. Materials and Methods: Adult patients consulting in the Retina Department of the Teaching Hospital of the National University of Asuncion, Paraguay between August and September, 2014 were included. Prior informed consent, socio-demographic and epidemiological data related to T. gondii infection were obtained. In addition a blood sample for the determination of anti $T$. gondii IgG antibodies by the ELISA method was taken and ophthalmologic evaluation for the diagnosis of OT was made. Results: A total of 80 patients with mean \pm SD age of $53 \pm 20$ years were studied, with slight predominance of women (55\%). The seroprevalence of toxoplasmosis was $84 \%(67 / 80)$ and OT was detected in $8.9 \%$ of the 67 seropositive persons. The habit of not washing vegetables with sodium hypochlorite and eat meat from wild animals was related to higher risk of infection in this population. Conclusion: It is important to conduct research at the population level to establish the epidemiology of toxoplasmosis in our country. Information on prophylactic measures to prevent infection by $T$. gondii should be given to the population.

Key words: Toxoplasmosis, seroprevalence, ocular toxoplasmosis, risk habits, Paraguay.

Palabras clave: Toxoplasmosis, seroprevalencia, toxoplasmosis ocular, hábitos de riesgo, Paraguay.

\section{Introducción}

L a toxoplasmosis, enfermedad que resulta de la infección por el protozoo Toxoplasma gondii, puede ser congénita o adquirida ${ }^{1}$. Este protozoo zoonótico infecta casi una tercera parte de la población mundial ${ }^{2-4}$. Las infecciones humanas pueden ocurrir fundamentalmente a través de tres rutas de transmisión: alimentaria (consumo de carne infectada por quistes tisulares), de animal a humano (ingestión de ooquistes liberados en las heces de gatos infectados) y de madre a feto (infección congénita, a través de la placenta durante el embarazo) ${ }^{1}$. Toxoplasma gondii puede también ser transmitido a través de transfusión sanguínea o trasplante de órganos de donantes infectados ${ }^{5-7}$. Una gran proporción de las infecciones adquiridas se relacionan a la ingestión de quistes en carnes crudas o mal cocidas y contactos con ooquistes esporulados en el ambiente ${ }^{5}$.

La toxoplasmosis aguda en personas inmunocompetentes es generalmente auto-limitada. Las manifestaciones de la infección por $T$. gondii son a menudo subclínicas, i.e. asintomática, o con síntomas no-específicos ${ }^{8}$, siendo los síntomas gripales o linfadenopatía las manifestaciones clínicas más comunes. La toxoplasmosis congénita puede causar lesión intracraneal y ocular en recién nacidos, que puede llevar a retardo mental y ceguera ${ }^{9}$. La manifestación clínica más común es la encefalitis; sin embargo, pueden presentarse también la corioretinitis, neumonía, o compromiso multi-orgánico9.

La toxoplasmosis ocular (TO), asociada con infecciones tanto congénitas como adquiridas, es una manifestación común de la enfermedad ${ }^{10}$; puede ocurrir en personas inmunocompetentes, ya sea de forma esporádica o en el contexto de un brote ${ }^{11}$. TO es la principal causa de uveítis posterior, una enfermedad que produce serias secuelas que incluyen pérdida completa de la visión ${ }^{12,13}$. Para pacientes inmunocomprometidos, la toxoplasmosis es una enfermedad que amenaza la vida. La carga de enfermedad por $T$. gondii y su potencial para causar brotes ${ }^{14}$ apoya la necesidad de identificar los factores de riesgo relacionados a esta enfermedad desatendida.

En Paraguay hay limitada información acerca de la toxoplasmosis en la población general. Un estudio reportó una muy alta prevalencia (94\%) de IgG anti T. gondii en una muestra pequeña de pacientes infectados con $\mathrm{VIH}^{15}$.

Varios estudios han investigado los factores de riesgo para toxoplasmosis congénita ${ }^{16}$; sin embargo, pocos estudios han evaluado los factores relacionados a la 
ocurrencia, severidad y recurrencia de TO. El objetivo de este estudio fue investigar la presencia de IgG anti $T$. gondii, factores de riesgo y la presencia de TO en pacientes que reciben atención oftalmológica en un Servicio de Oftalmología.

\section{Pacientes y Métodos}

Se realizó un estudio observacional, descriptivo, de corte transversal. Fueron invitados a participar del estudio pacientes que acudieron a consultar a la Cátedra de Oftalmología del Hospital de Clínicas entre agosto y septiembre del año 2014 por problemas de visión. A cada participante se le pidió que firmara un consentimiento informado una vez que se les explicó detalladamente los objetivos y procedimientos del estudio. El protocolo fue aprobado por los comités Científico y Ético del Instituto de Investigaciones en Ciencias de la Salud.

Los pacientes fueron entrevistados por un encuestador entrenado quien completaba un cuestionario con los datos personales del paciente como edad, sexo, procedencia, además de datos clínicos y epidemiológicos que incluía hábitos de riesgo para adquirir toxoplasmosis. Los datos clínicos registrados fueron: presencia de síntomas como manifestaciones oculares, cefalea, dolor abdominal, convulsiones. Se les interrogó además, por hábitos como consumo de carne de cerdo, vacuna, pollo no bien cocinado o vegetales, tipo de agua de consumo, contacto con mascotas (perros y gatos).

Posteriormente se procedió a la toma de una muestra de sangre venosa sin anticoagulante para la determinación serológica de IgG anti-Toxoplasma gondii. Las muestras de suero fueron almacenadas a $-20^{\circ} \mathrm{C}$ hasta su procesamiento.

La presencia de $\operatorname{IgG}$ anti $T$. gondii fue determinada por el método de ELISA indirecto IgG (TOXO-TEST IICSParaguay). El kit consta de microplacas sensibilizadas con un antígeno obtenido de taquizoitos de la cepa RH de $T$. gondii. Durante el primer paso de incubación los anticuerpos anti- $T$. gondii presentes en la muestra se ligan con el antígeno fijado en la microplaca. Después de haber eliminado por lavado los componentes séricos no reactivos, en el segundo paso de incubación, los anticuerpos humanos fijados reaccionan con un anticuerpo anti-IgG humano, conjugado con peroxidasa (POD). La concentración de la enzima peroxidasa en el complejo formado se revela con el sustrato. La intensidad del color es directamente proporcional a la concentración de anticuerpos anti $T$. gondii presentes en la muestra.

El diagnóstico de toxoplasmosis ocular fue realizado por oftalmólogos experimentados utilizando un oftalmoscopio binocular indirecto y en aquellos casos con sospecha de enfermedad activa se realizó un examen con lámpara de hendidura. Se consideró que eran focos activos de corio-retinitis la presencia de reacción de cámara anterior y/o flare, precipitados queráticos, asociado a la presencia células en el vítreo, opacidades vítreas o vitreítis franca con lesiones amarillentas o blanquecinas sobre-elevadas y mal delimitadas, de tipo algodonoso y exudativo, rodeado de una zona hiperémica con vasculitis perilesional y vasos envainados. Las lesiones inactivas de corio-retinitis, consideradas como cicatrices de toxoplasmosis se presentan como áreas de atrofia blanquecinas en el centro, con bordes pigmentados y bien delimitados, sin signos de inflamación perilesional.

Los datos fueron introducidos en una hoja de cálculo de Excel y posteriormente analizados usando el software Epi-Info 3.1 (CDC, Atlanta, USA). Se utilizó estadística descriptiva expresando los resultados en forma de distribución de frecuencia para las variables nominales $\mathrm{y}$ medidas de tendencia central y dispersión para las variables continuas. Para indagar posibles asociaciones fueron usadas las pruebas de chi cuadrado o prueba exacta de Fisher y la fuerza de la asociación expresada en forma de odds ratio (OR); los promedios de edades fueron comparadas usando la prueba t de Student, todas a un nivel de significancia de 0,05 .

\section{Resultados}

Fueron incluidos en el estudio 80 pacientes, con un leve predominio del sexo femenino (55\%). Se observó mayor frecuencia de pacientes mayores de 60 años de edad $(43,8 \%)$ y proveniente del Departamento Central (63\%). Más de la mitad (56\%) tenía como fuente de agua de consumo el servicio de abastecimiento público. El principal motivo de consulta fue la disminución de la visión $(65 / 80 ; 81 \%)$; cada paciente presentó más de un síntoma clínico (Tabla 1 ).

Un alto porcentaje de los pacientes $(67 / 80 ; 84 \%)$ presentó serología positiva para $T$. gondii. De los 67 pacientes con serología positiva, seis $(8,9 \%)$ presentaron $\mathrm{TO}$, de los cuales cuatro pacientes tenían foco activo y dos cicatrices.

Con respecto a los conocimientos sobre la toxoplasmosis, $41 \%$ reportó conocer la enfermedad, menor proporción reportó conocer el modo de transmisión (33\%).

En relación a los hábitos de riesgo para adquirir toxoplasmosis se observó que $86,3 \%$ tenía alguna mascota siendo el perro $(66,2 \%)$ y el gato $(33,8 \%)$ los más frecuentes; entre ellos, $70 \%$ refería dormir con su mascota. El consumo de verduras crudas se observó en $87,5 \%$, consumo de carne de cerdo en $65 \%$ y de carne silvestre en 53,8\% (Tabla 2).

No se observaron diferencias significativas entre los seropositivos y seronegativos con respecto al sexo (valor $\mathrm{p}: 0,127$ ), grupo etario (valor $\mathrm{p}: 0,710$ ) ni procedencia (valor p: 0,351). Tampoco los síntomas en el momento de consulta arrojaron diferencias significativas (Tabla 3 ). 


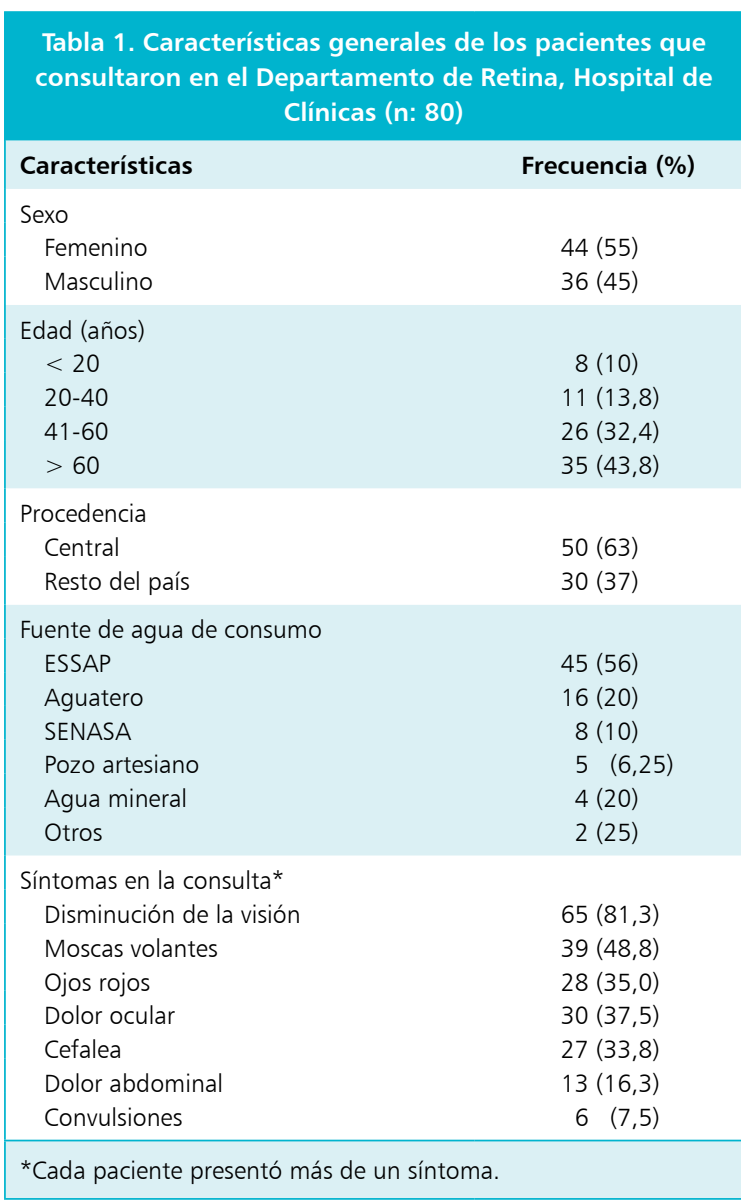

Tabla 3. Seropositividad para Toxoplasma gonditi de acuerdo a las características clínicas y socio-demográficas de los pacientes ( $\mathrm{n}: \mathbf{8 0}$ )

\begin{tabular}{lrc}
\hline Características & Seropositividad & Valor $\mathbf{p}$ \\
Sexo & & 0,127 \\
$\quad$ Femenino & $34 / 44(50,7)$ & \\
$\quad$ Masculino & $33 / 36(49,3)$ & \\
Edad (años) & & 0,710 \\
$\quad<20$ & $7 / 8(10,4)$ & \\
$20-40$ & $8 / 11(11,9)$ & \\
$41-60$ & $22 / 26(32,8)$ & \\
$>60$ & $30 / 35(44,7)$ & \\
Procedencia & & 0,351 \\
$\quad$ Departamento central & $40 / 50(59,7)$ & \\
$\quad$ Resto del país & $27 / 30(40,3)$ & \\
Síntomas en la consulta & & \\
$\quad$ Disminución de la visión & $54(81)$ & 0,734 \\
Moscas volantes & $34(36)$ & 0,417 \\
Ojos rojos & $24(36)$ & 0,727 \\
Dolor ocular & $23(34)$ & 0.183 \\
Cefalea & $23(34)$ & 0,804 \\
Dolor abdominal & $11(16)$ & 0,926 \\
Convulsiones & $5(7,4)$ & 0,977 \\
\hline
\end{tabular}

Tabla 2. Seroprevalencia de Toxoplasma gondit, conocimientos de la enfermedad y hábitos de riesgo para adquirirla

\begin{tabular}{|lc|}
\hline Características & Frecuencia (\%) \\
Serología positiva (anti IgG T. gondii) & $67(84)$ \\
Toxoplasmosis ocular & $6 / 67(8,9)$ \\
Conocimiento sobre toxoplasmosis & \\
Escuchó sobre la enfermedad & $33(41)$ \\
Conoce el modo de transmisión & $26(33)$ \\
Conoce el agente transmisor & $16(20)$ \\
Conoce la manifestaciones clínicas & $11(13,8)$ \\
Tuvo diagnóstico anterior para toxoplasmosis & $6(7,5)$ \\
Hábitos de riesgo para adquirir toxoplasmosis & \\
Come verduras crudas & $70(87,5)$ \\
Tiene mascota & $69(86,3)$ \\
Duerme con su mascota & $56(70,0)$ \\
Consume carne de cerdo & $52(65,0)$ \\
Consume carne silvestre & $43(53,8)$ \\
Consume carnes semi-crudas & $16(20,0)$ \\
Lava verduras con hipoclorito de sodio & $12(15,0)$ \\
Mascotas & $6(7,5)$ \\
Perro & $10(12,5)$ \\
Gato & \\
Gato y perro & $37(46,2)$ \\
Otras & $11(13,8)$ \\
Ninguno & $16(20)$ \\
\hline
\end{tabular}

En relación a los hábitos de riesgo para adquirir toxoplasmosis, dos factores se relacionaron con la serología positiva: no lavar las verduras con hipoclorito de sodio $\left(\mathrm{RR}=3,6 \mathrm{IC}_{95 \%} 1: 1,3-9,8\right)$ y comer carne silvestre $\left(\mathrm{RR}=4,9 ; \mathrm{IC}_{95 \%} \% 1,2-19,6\right)$. Los otros hábitos no arrojaron asociaciones con adquirir la infección (Tabla 4).

Las características demográficas y clínicas de los pacientes con TO fueron similares a los pacientes seropositivos sin afectación ocular (Tabla 5).

\section{Discusión}

La frecuencia de toxoplasmosis encontrada en la población paraguaya estudiada fue de $84 \%$, siendo mayor en personas mayores de 60 años de edad. Este resultado era de esperar considerando que la prevalencia de infección con T. gondii en el hombre es elevada en todo el mundo.

Las características del medio influyen en la prevalencia, siendo mayor en regiones cálidas y/o húmedas, y más baja en climas secos y fríos ${ }^{2}$. También hay diferencias en las tasas de positividad con relación a la altitud, correspondiendo las más altas a las áreas de mayor elevación sobre el nivel del mar. Países como Bélgica reportan una positividad de $16,9 \%$ en personas mayores de 30 años, Países Bajos presenta una tasa de $64 \%$ entre la población 
de 20 a 22 años y Estados Unidos de América alcanza hasta $67 \%$ en individuos mayores de 50 años. En América Central, Francia, Turquía y Brasil, la seroprevalencia es mucho mayor (aproximadamente 90\%) alrededor de los 40 años. Todos estos datos demuestran que la positividad aumenta con la edad, y que la infección existe en todos los países en que ha sido estudiada ${ }^{8}$.

Aparentemente, los factores económicos y sociales no tienen relación especial con el parásito; sin embargo, sí los factores culturales, pues la costumbre de comer carne cruda o mal cocinada y la de tener contacto con gatos en los hogares aumentan la probabilidad de infección ${ }^{2,4}$. En nuestro estudio encontramos algunos factores de riesgo, como la mala higiene de los alimentos evidenciada por la falta de lavado de las frutas y verduras con hipoclorito de sodio, recomendado en nuestro país por la alta frecuencia de parasitosis intestinal, y suponemos que la práctica sería también eficiente para eliminar los ooquistes de $T$. gondii, además del consumo de carne de animales silvestres en muchos casos insuficientemente cocidas. No hemos evaluado el número de tenencia de mascotas o factores laborales de riesgo para adquirir toxoplasmosis como ser carnicero, jardinero, entre otras.

Se incluyeron preguntas en relación al conocimiento de la enfermedad debido a que en algunos estudios se han demostrado relación con los hábitos higiénicos, muy importantes en la transmisión de la toxoplasmosis ${ }^{17-19}$; sin embargo, no encontramos relación con los mismos, probablemente debido a que la población que consulta en este hospital es muy homogénea.

Por otro lado, se consideraron síntomas como cefalea, dolor ocular, dolor abdominal, como una forma de establecer infecciones recientes de la enfermedad; no obstante, no pudimos establecer relación con la presencia de los mismos.

De los 67 pacientes seropositivos, 8,9\% presentó lesiones compatibles con TO como foco activo (4/6) o cicatrices de corio-retinitis (2/6). Esta frecuencia es similar a las reportadas en otros países como Brasil, la que varía entre 5,6 y $30 \%$ en pacientes con problemas de visión. Sin embargo, en Alabama, E.U.A., la prevalencia fue menor a $1 \%{ }^{20-22}$. La prevalencia de TO varía en diferentes regiones alrededor del mundo; en Brasil alrededor de 30\% de los pacientes con enfermedad ocular tiene $\mathrm{TO}^{23,24}$. En los estudiantes de la ciudad de Natal (RN), la prevalencia encontrada fue de 1,2\%, y en Erichim al sur de Brasil fue de $17,7 \%{ }^{25-28}$. Otros trabajos en Brasil demostraron diferencias en las prevalencias dependiendo del área geográfica, condiciones ambientales y franja etaria estudiada ${ }^{29,30}$. En un estudio poblacional en un barrio de Río de Janeiro-Brasil, la prevalencia de las lesiones cicatrizadas compatibles con TO fue de $3,8 \%$ en 1.071 individuos y 5,8\% en los individuos con serología positiva para $T$. gondii, y en un estudio en un Servicio de Baja Visión de Brasil, la etiología más

\begin{tabular}{|c|c|c|c|}
\hline & \multicolumn{2}{|c|}{ Serología (IgG) } & \multirow[b]{2}{*}{ Valor $p$} \\
\hline & Positiva $(n=67)$ & Negativa $(n=13)$ & \\
\hline \multicolumn{4}{|l|}{ Conocimientos } \\
\hline Escuchó sobre la toxoplasmosis & $25(37 \%)$ & $8(62 \%)$ & 0,104 \\
\hline Modo de transmisión & $21(31 \%)$ & $5(38 \%)$ & 0,616 \\
\hline Agente transmisor & $12(18 \%)$ & $4 \quad(31 \%)$ & 0,289 \\
\hline Manifestaciones clínicas & $7(10 \%)$ & $4 \quad(31 \%)$ & 0,073 \\
\hline \multicolumn{4}{|l|}{ Hábitos } \\
\hline Lava verduras con hipoclorito de sodio & $7(10 \%)$ & $5(38 \%)$ & 0,010 \\
\hline Come verduras crudas & $58(87 \%)$ & 12 (92\%) & 0,567 \\
\hline Consume carnes semi-crudas & $15(22 \%)$ & $1 \quad(7,6 \%)$ & 0,225 \\
\hline Consumen carne de cerdo & $45(68 \%)$ & $7 \quad(54 \%)$ & 0,549 \\
\hline Consume carne silvestre & $40(60 \%)$ & $3(23 \%)$ & 0,015 \\
\hline Tiene mascotas & $56(84 \%)$ & $13(100 \%)$ & 0,083 \\
\hline Duerme con sus mascotas & $54(81 \%)$ & $2(15 \%)$ & 0,355 \\
\hline
\end{tabular}

Tabla 5. Características demográficas, manifestaciones clínicas y factores de riesgo en pacientes con toxoplasmosis ocular

\begin{tabular}{|lcc|}
\hline Características & $\begin{array}{c}\text { Con To } \\
\text { Frecuencia (n: 6) }\end{array}$ & $\begin{array}{c}\text { Sin TO } \\
\text { Frecuencia (n: 61) }\end{array}$ \\
$\begin{array}{l}\text { Sexo } \\
\text { Femenino }\end{array}$ & \\
$\quad$ Masculino & $1(16,7 \%)$ & $33(54 \%)$ \\
Edad (años) & $5(83,3)$ & $28(46 \%)$ \\
$\quad<20$ & & \\
$20-40$ & $1(16,7 \%)$ & $6(9,8)$ \\
$41-60$ & $2(33,3 \%)$ & $6(9,8)$ \\
$>60$ & $1(16,7 \%)$ & $21(34,4)$ \\
Hábitos & $2(33,3 \%)$ & $28(45,9)$ \\
Come verduras crudas & & \\
Consume carne de cerdo & $5(83,3 \%)$ & $53(86,9)$ \\
Tiene mascotas & $5(83,3 \%)$ & $40(65,6)$ \\
Consume carnes semi-crudas & $4(66,7 \%)$ & $52(85,2)$ \\
Consume carne silvestre & $1(16,7 \%)$ & $14(23,0)$ \\
Duerme con sus mascotas & $1(16,7 \%)$ & $39(63,9)$ \\
Lava verduras con hipoclorito de sodio & $1(16,7 \%)$ & $53(86,9)$ \\
\hline TO: toxoplasmosis ocular. & 0 & $7(11,5)$ \\
\hline
\end{tabular}

frecuente en pacientes entre 20-39 años de edad fue TO $(21,1 \%)$ y la prevalencia global fue $10 \%{ }^{22}$. Sin embargo, en E.U.A., la prevalencia de lesiones de corio-retinitis sugerentes de toxoplasmosis en la población general en Maryland, Alabama, fue de $0,6 \%{ }^{20,21}$. Esto podría deberse al clima más frío en ese país en comparación a Paraguay que es más cálido y húmedo².

Considerando al sexo como un factor predisponente para adquirir toxoplasmosis, no hemos encontrado una diferencia significativa en la serología positiva para toxoplasmosis. En nuestro estudio, tuvimos un marcado 
predominio de TO en los varones $83 \%$ (5/6) y con factores de riesgo como comer verduras crudas, carne de cerdo y tener mascotas. Según el estudio de Akstein y cols..$^{31}$, la TO se presenta en un amplio rango de edades, que coincide con nuestro estudio; no obstante, se observó mayor afectación en los pacientes entre 20 y 40 años.

No existen pruebas concluyentes de que la toxoplasmosis tenga preferencia por un sexo, aunque algunos trabajos encuentran cifras superiores en hombres que en mujeres ${ }^{32}$ lo que coincide con los resultados obtenidos en nuestra serie. Según algunos autores, la TO es más frecuente en los hombres debido a sus ocupaciones: campesinos, labradores, ganaderos, trabajadores de granjas avícolas y mataderos, cocineros, curtidores de piel, veterinarios, entre otras, donde están más expuestos ${ }^{33,34}$.

Una limitación del estudio es la muestra sesgada, dado que los pacientes que consultan a una clínica oftalmológica pueden tener mayor seroprevalencia, debido a la mayor probabilidad de encontrar pacientes con TO y, por lo tanto, puede no ser representativa de la población general.

Otro punto importante que se puede mencionar es que el estudio tuvo carácter de tamizaje, considerando el uso de la serología para toxoplasmosis con IgG y una sola técnica, pues en general, se acepta que deben existir dos técnicas para considerar un paciente positivo. Tampoco se tituló la serología.

En conclusión, hemos encontrado una alta frecuencia de toxoplasmosis en pacientes que acuden al Servicio de Oftalmología en Paraguay; la prevalencia de TO estuvo dentro del rango publicado a nivel internacional, con un predominio del sexo masculino y con un rango muy amplio de edad. La presencia de animales domésticos, los hábitos alimentarios no adecuados y falta de higiene son factores de riesgo importantes en la epidemiología de esta parasitosis en nuestro país. Es importante realizar trabajos de investigación en nuestra población para establecer la epidemiología de la toxoplasmosis en el país. Se debe dar a conocer a la población las medidas de profilaxis para evitar la infección por T. gondii.

\section{Resumen}

Introducción: La toxoplasmosis es una enfermedad de distribución mundial, que puede ocasionar disminución de la visión hasta ceguera. La vía de transmisión en el hombre puede variar de acuerdo a los hábitos de cada región, siendo probablemente la ingestión de carne cruda o mal cocida la principal vía de contagio. Objetivo: Determinar la seroprevalencia de toxoplasmosis en una clínica oftalmológica, la frecuencia de toxoplasmosis ocular (TO) y los hábitos de riesgo para adquirir la enfermedad. Pacientes y Métodos: Fueron incluidos 80 pacientes adultos que consultaron en el Departamento de Retina de la Cátedra de Oftalmología del Hospital de Clínicas entre agosto y septiembre de 2014. Previo consentimiento informado, se obtuvieron los datos socio-demográficos y epidemiológicos relacionados a la infección por Toxoplasma gondii. Además se tomó una muestra de sangre para la determinación de anticuerpos del tipo IgG anti $T$. gondii por el método de ELISA y se realizó la evaluación oftalmológica para el diagnóstico de TO. Resultados: La edad promedio \pm DE fue de 53 \pm 20 años, con leve predominio de mujeres (55\%). La seroprevalencia de toxoplasmosis fue de $84 \%(67 / 80)$ y la TO se detectó en $8,9 \%$ de los 67 seropositivos. Se observó que el hábito de no lavar las verduras con hipoclorito de sodio y comer carne silvestre presentó mayor riesgo de contraer la infección en esta población. Conclusión: Es importante realizar trabajos de investigación a nivel poblacional para establecer la epidemiología de la toxoplasmosis en nuestro país. Se debe dar a conocer a la población las medidas de profilaxis para evitar la infección por $T$. gondii.

\section{Referencias bibliográficas}

1.- Hill D, Dubey J P. Toxoplasma gondii: transmission, diagnosis and prevention. Clin Microbiol Infect 2002; 8 (10): 634-40.

2.- Hill D E, Chirukandoth S, Dubey J P. Biology and epidemiology of Toxoplasma gondii in man and animals. Anim Health Res Rev 2005; 6: 41-61.

3.- Montoya J G, Liesenfeld O. Toxoplasmosis. Lancet 2004; 363: 1965-76.

4.- Pappas G, Roussos N, Falagas M E. Toxoplasmosis snapshots: global status of Toxoplasma gondii seroprevalence and implications for pregnancy and congenital toxoplasmosis. Int J Parasitol 2009; 39:
1385-94.

5.- Remington J S, McLeod R, Desmonts G. Toxoplasmosis. In: Remington JS, Klein JO, editors. Infectious Diseases of the Fetus and Newborn. 5th ed. Philadelphia: W.B. Saunders; 2001. p. 205-346.

6.- Siegel S E, Lunde M N, Gelderman A H, Halterman R H, Brown J A, Levine A S, et al. Transmission of toxoplasmosis by leukocyte transfusion. Blood 1971; 37: 388-94.

7.- Derouin F, Pelloux H. Prevention of toxoplasmosis in transplant patients. Clin Microbiol Infect 2008; 14: 1089-101.

8.- Antoniazzi E, Guagliano R, Meroni V, Pezzotta S, Bianchi P E. Ocular impairment of toxoplasmosis. Parassitologia 2008; 50 (1-2): 35-6.
9.- Havelaar A H, Kemmeren J M, Kortbeek L M. Disease burden of congenital toxoplasmosis. Clin Infect Dis 2007; 44: 1467-74.

10.- Weiss L M, Dubey J P. Toxoplasmosis: A history of clinical observations. Int J Parasitol 2009; 39 (8): 895-901.

11.- Bowie W R, King A S, Werker D H, IsaacRenton J L, Bell A, Eng S B, et al. Outbreak of toxoplasmosis associated with municipal drinking water. Lancet 1997; 350: 173-7.

12.- Hay J, Dutton G N. Toxoplasma and the eye. Br Med J 1995; 310: 1021-2.

13.- Fernandes L C, Orefice F. Aspectos clínicos e epidemiológicos das uveítes em serviço de referência em Belo Horizonte 1970-1993. Rev Bras Oftalmol 1995; 55: 569-78.

14.- Dubey J P. Toxoplasmosis-a waterborne 
zoonosis. Vet Parasitol 2004; 126: 57-72.

15.- San Miguel C, Ferreira J, Figueredo N, Fleitas D, Canese A. Prevalencia de Toxoplasmosis y Chagas en pacientes con VIH/SIDA. Rev Inst Med Trop 2010; 5 (2): 10-5.

16.- Spalding S M, Amendoeira M R R, Klein C H, Ribeiro L C. Serological screening and toxoplasmosis exposure factors among pregnant women in South of Brazil. Rev Soc Bras Med Trop 2005; 38 (2): 173-7.

17.- Kamani J, Mani A U, Egwu G O, Kumshe H A. Seroprevalence of human infection with Toxoplasma gondii and the associated risk factors, in Maiduguri, Borno state, Nigeria. Ann Trop Med Parasitol 2009; 103: 317-21.

18.- Fan C K, Lee L W, Liao C W, Huang Y C, Lee Y L, Chang Y T, et al. Toxoplasma gondii infection: relationship between seroprevalence and risk factors among primary schoolchildren in the capital areas of Democratic Republic of São Tome and Principe, West Africa. Parasit Vectors 2012; 5: 141.

19.- Fu C J, Chuang T W, Lin H S, Wu C H, Liu Y $\mathrm{C}$, Langinlur M K, et al. Toxoplasma gondii infection: seroprevalence and associated risk factors among primary school children in the capital area of the Republic of the Marshall Islands. Jpn J Infect Dis 2014; 67: 405-10.

20.- Maetz H M, Kleinstein R N, Federico D, Wayne $\mathrm{J}$. Estimated prevalence of ocular toxoplasmosis and toxocariasis in Alabama. J Infect Dis 1987
Aug; 156 (2): 414.

21.- Smith R E, Ganley J P. Ophthalmic survey of a community. 1. Abnormalities of the ocular fundus. Am J Ophthalmol 1972; 74 (6): 112630.

22.- Silva A M T, Matos M H B R, Lima H C. Serviço de visão subnormal do Instituto Brasileiro de Oftalmologia e Prevenção da Cegueira (IBOPC): análise dos pacientes atendidos no $1^{\circ}$ ano do departamento (2004). Arq. Bras. Oftalmol. 2010; 73 (3): 266-70.

23.- Matos K, Muccioli C, Belfort Junior R, Rizzo L V. Correlation between clinical diagnosis and PCR analysis of serum, aqueous, and vitreous samples in patients with inflammatory eye disease. Arq Bras Oftalmol. 2007; 70 (1): 10914.

24.- Arévalo J F, Belfort R Jr, Muccioli C, Espinoza $\mathrm{J}$ V. Ocular toxoplasmosis in the developing world. Int Ophthalmol Clin. 2010 Spring; 50 (2): 57-69.

25.- de Amorim García C A, Oréfice F, de Oliveira Lyra C, Gomes A B, França M, de Amorim Garcia Filho CA. Socioeconomic conditions as determining factors in the prevalence of systemic and ocular toxoplasmosis in Northeastern Brazil. Ophthalmic Epidemiol 2004; 11 (4): 301-17.

26.- Glasner P D, Silveira C, Kruszon-Moran D, Martins M C, Burnier Júnior M, Silveira S, et al. An unusually high prevalence of ocular toxoplasmosis in southern Brazil. Am J
Ophthalmol 1992; 114 (2): 136-44.

27.- Melamed J. Peculiaridades da toxoplasmose ocular no Rio Grande do Sul. Arq Bras Oftalmol 1988; 51: 197-8.

28.- Silveira C, Belfort Jr R, Burnier Jr M, Nussemblat R. Acquired toxoplasmic infection as the cause of retinocoroidititis in families. Am J Ophthalmol 1988; 196: 362-4.

29.- Abreu M T, Belfort Jr R, García A R, Muccioli C, Soriano E, Nussenblat R, et al. Toxoplasmose ocular em Venda Nova do Imigrante, ES, Brasil. Arq Bras Oftalmol 1998; 61: 540-5.

30.- Amendoeira M R, Sobral C A, Teva A, de Lima J N, Klein CH. Serological survey of Toxoplasma gondii infection in isolated Amerindians, Mato Grosso. Rev Soc Bras Med Trop 2003; 36 (6): 671-6.

31.- Akstein R B, Wilson L A, Teutsch S M. Acquired toxoplasmosis. Ophthalmology 1982; 89 (12): 1299-302.

32.- Toledo González Y, Soto García M, Chiang Rodríguez C, Rúa Martínez R, Estévez Miranda Y, Santana Alas E R. Comportamiento clínicoepidemiológico de la toxoplasmosis ocular. Rev Cubana Oftalmol 2011; 24 (1): 15-29.

33.- Pardo A, Collizo J, Valldeperas X. Revisión de la prevención y tratamiento de la toxoplasmosis ocular. Ann Oftalmol 2004; 12 (1): 11-20.

34.- Holland G N. Ocular toxoplasmosis: a global reassessment. Part I: epidemiology and course of disease. Am J Ophthalmol 2003; 136 (6): 973-88. 